\section{Cobertura estimada da triagem auditiva neonatal para usuários do Sistema Único de Saúde, Brasil, 2008-2011}

\section{Estimated coverage of newborn hearing screening among users of the Brazilian National Health System, 2008-2011}

Lorena Rauédys Leite da Cruz 1 Silvia Ferrite 2

\begin{abstract}
Objectives: to estimate and describe the coverage of newborn hearing screening (NHS) among users of the Brazilian National Health System (SUS) between 2008 and 2011.

Methods: an ecological study of time series was conducted using data from the SUS's Outpatients Information System (SIA-SUS), the Live Births Information System (SINASC), the Interagency Health Information Network (RIPSA) and the Beneficiaries Information System (SIB) of the National Supplementary Health Agency (ANS). Coverage was estimated based on the NHS procedures authorized by the SUS and the live newborns are users of SUS, for every year for each federal unit.

Results: national NHS coverage among SUS users was estimated to have been $7.1 \%$ in 2008, and rose to $21.8 \%$ in 2011, with evidence disparities between and within regions. More extensive coverage was found in the states of Rio Grande do Sul (60.1\%) and Paraná (59.4\%), while, in the states of Rondonia, Espirito Santo and Pernambuco, coverage was less than 5\%.

Conclusions: in Brazil, more than two thirds of newborns who are users of the SUS did not undergo hearing screening, even in 2011, the following year the passing of a law making the procedure obligatory. Although there has been progress, the goal should be universal coverage.
\end{abstract}

Key words Neonatal screening, State health care coverage, Unified Health System
1 Residência Integrada Multiprofissional em Saúde. Complexo Hospitalar Universitário Professor Edgard Santos. Universidade Federal da Bahia. Salvador, BA, Brasil.

2 Departamento de Fonoaudiologia. Instituto de Ciências da Saúde. Universidade Federal da Bahia. Av. Reitor Miguel Calmon, s.n. Vale do Canela. Salvador, BA, Brasil. CEP: 40.110-902.

E-mail: ferrite@ufba.br

\section{Resumo}

Objetivos: estimar e descrever a cobertura da triagem auditiva neonatal (TAN) para os usuários do Sistema Único de Saúde (SUS) no Brasil, entre 2008 e 2011.

Métodos: estudo ecológico de séries temporais utilizando-se dados do Sistema de Informação Ambulatorial do SUS (SIA-SUS), Sistema de Informações sobre Nascidos Vivos (SINASC), Rede Interagencial de Informações para a Saúde (RIPSA) e Sistema de Informações de Beneficiários (SIB) da Agência Nacional de Saúde Suplementar (ANS). A cobertura foi estimada considerando-se os procedimentos para TAN autorizados pelo SUS e os nascidos vivos usuários do SUS, para cada ano e Unidade da Federação.

Resultados: a cobertura nacional da TAN para usuários do SUS foi estimada em 7,1\% em 2008, e alcançou 21,8\% em 2011, com evidências de desigualdades inter e intrarregionais. Maiores coberturas foram observadas no Rio Grande do Sul $(60,1 \%)$ e no Paraná $(59,4 \%)$, enquanto Rondônia, Espírito Santo e Pernambuco apresentaram cobertura inferior a $5 \%$.

Conclusões: no país, mais de dois terços dos neonatos usuários do SUS não foram submetidos à triagem auditiva, mesmo em 2011, ano seguinte à promulgação da lei nacional que estabeleceu a obrigatoriedade do procedimento. Embora o cenário seja de avanço, a meta é a cobertura universal.

Palavras-chave Triagem neonatal, Cobertura de serviços públicos de saúde, Sistema Único de Saúde 


\section{Introdução}

Estimativas indicam que, a cada 1000 nascidos vivos, entre um a seis apresentam algum grau de deficiência auditiva. ${ }^{1}$ A privação sensorial da audição nos primeiros anos de vida prejudica o processo de aquisição e desenvolvimento da linguagem oral, o que poderá interferir no desenvolvimento social, psíquico e educacional desse indivíduo, assim como na dinâmica familiar, e futuramente no seu percurso acadêmico e laboral.2,3 $\mathrm{O}$ diagnóstico precoce é essencial para que a intervenção ocorra em tempo hábil para evitar ou minimizar as consequências negativas à saúde e à qualidade de vida dos indivíduos com deficiência auditiva. 4 Desta forma, em vista da magnitude do problema na população, dos prejuízos linguísticos, educacionais e psicossociais decorrentes, dos custos sociais, e da importância do diagnóstico e intervenção precoces e, ainda, considerando-se o potencial de prevenção, a deficiência auditiva na infância configura-se como um problema de saúde pública.

Embora a incidência da deficiência auditiva seja maior entre os neonatos com indicadores de risco específicos - determinadas infecções congênitas, síndromes genéticas, anomalias craniofaciais, permanência em Unidades de Terapia Intensiva, histórico familiar de surdez congênita, entre outros aproximadamente $50 \%$ dos casos não apresentam tais indicadores em sua história. 5 Assim, justifica-se a necessidade de que estratégias de detecção da deficiência auditiva ao nascimento sejam universais, e não restritas aos neonatos classificados em grupos de alto risco. Esta é a premissa da Triagem Auditiva Neonatal Universal (TANU), que permite a detecção precoce, com procedimento de alta sensibilidade, para que a intervenção possa ser iniciada antes dos seis meses de vida da criança, possibilitando melhores resultados no desenvolvimento da função auditiva, da linguagem, da fala, do processo de aprendizagem, e consequentemente melhor inclusão social. Diante disso, a TANU faz parte de um conjunto de ações que devem ser realizadas visando a atenção integral à saúde na infância. ${ }^{6}$ Nesse sentido, em diversos países, as políticas públicas de saúde têm preconizado e desenvolvido Programas de Triagem Auditiva Neonatal Universal (PTANU). ${ }^{?}$

No Brasil, o acesso à Triagem Auditiva Neonatal (TAN), em caráter universal ou restrito ao grupo de alto risco, concentra-se nas maternidades privadas e/ou no nível ambulatorial via assistência suplementar, sendo mais raro nas maternidades e serviços públicos. $8 \mathrm{O}$ conceito de acesso está fortemente ligado à justiça social, e assim, comumente reflete questões relacionadas às desigualdades sociais, que devem ser superadas por meio de ações que visem a equidade, tida como uma das prioridades para o Ministério da Saúde.9,10 Visando a melhoria do acesso aos serviços de saúde e a integralidade da assistência à saúde na infância, em 2010, a Lei 12.303 tornou obrigatória a realização de um procedimento de triagem, denominado emissões otoacústicas evocadas (EOA), em todas as maternidades e hospitais, gratuitamente, para a totalidade das crianças nascidas em suas dependências. ${ }^{11}$

Impulsionado pela promulgação da Lei, esperase que o quadro atual evolua positivamente, especialmente para os usuários do Sistema Único de Saúde (SUS). Para o monitoramento da situação nacional da triagem auditiva neonatal, e posterior verificação do impacto da Lei, são necessários dados de vigilância, que sirvam como balizadores para a avaliação da implementação da TANU no Brasil. Porém, não foram identificadas publicações sobre a cobertura da TAN no país.

Diante desse contexto, o objetivo deste estudo foi estimar a cobertura da TAN para usuários do SUS no Brasil, identificar as diferenças regionais e o crescimento da cobertura, no período de 2008 a 2011.

\section{Métodos}

Neste estudo de vigilância, a população de referência foram os nascidos vivos no país, usuários do SUS, no período de 2008 a 2011. Trata-se de estudo ecológico descritivo de séries temporais, no qual foram tomadas como unidades de análise as Unidades da Federação (UF) do Brasil. Foram utilizados como fonte de dados: o Sistema de Informação Ambulatorial do SUS (SIA-SUS), o Sistema de Informações sobre Nascidos Vivos (SINASC), a Rede Interagencial de Informações para a Saúde (RIPSA) e o Sistema de Informações de Beneficiários (SIB) da Agência Nacional de Saúde Suplementar (ANS).

No SIA-SUS foi obtido o número de procedimentos de "emissões otoacústicas evocadas para triagem auditiva", código $\mathrm{n}^{\circ}$ 02.11.07.014-9, aprovados pelo SUS, para cada ano e UF de atendimento entre 2008 e 2011. No SINASC, foi identificado o número de nascidos vivos por ano e UF no mesmo período. Por motivo de sub-registro, a cobertura do SINASC vem sendo monitorada, e anualmente a RIPSA divulga dados que permitem a sua correção, especificamente, a razão entre os nascidos vivos informados e estimados por UF. Assim, para evitar vieses, o número corrigido de nascidos vivos 
(NV) por UF foi calculado utilizando-se a fórmula: $\mathrm{NV}_{\text {corrigido }}=(\mathrm{NV} / \mathrm{FC}) \times 100$, onde $\mathrm{NV}$ é o número de nascidos vivos obtido no SINASC, e FC é o Fator de Correção da RIPSA que corresponde à razão entre os nascidos vivos informados e estimados, por UF e ano. No SIB/ANS, foi identificada a cobertura total da assistência suplementar de saúde, por UF e ano, para o cálculo da parcela da população usuária do SUS, devido à grande variação no território nacional. Assim, a cobertura da TAN para nascidos vivos usuários SUS foi estimada para cada ano-calendário, para o país e por UF, considerando-se a seguinte fórmula: Cobertura $=$ nTA $\times 100 /$ NVSUS, onde nTA corresponde ao número de EOA para triagem auditiva aprovadas pelo SUS por UF e ano, e NVSUS corresponde ao número de nascidos vivos usuários do SUS. Especificamente, a estimativa de NVSUS foi calculada utilizando-se a parcela complementar da cobertura total da assistência suplementar (CAS) para cada UF e ano, utilizandose a fórmula: NVSUS $=\mathrm{NV}_{\text {cor }} \times[(100-\mathrm{CAS}) / 100]$, onde $\mathrm{NV}_{\text {cor }}$ é o número de nascidos vivos corrigido, e CAS é o percentual correspondente à cobertura da assistência suplementar de saúde.

Para a estimativa da cobertura por região geográfica, para cada ano calendário, foram considerados os somatórios dos procedimentos e dos NVSUS correspondentes às UF de cada região: Cobertura $_{\text {REGIÃO }}=\sum$ nTA x $100 / \sum$ NVSUS. Para a análise do crescimento da cobertura da TAN para usuários do SUS, foi calculada a variação proporcional percentual (VPP) no período 2008-2011, para o país e regiões geográficas, utilizando-se a fórmula: $\mathrm{VPP}=[($ Cobertura2011 - Cobertura2008) $/$ Cobertura2008] x 100. As análises foram realizadas utilizando-se o programa Excel $^{\circledR}$, e os mapas foram elaborados utilizando-se o TABWIN, versão 32.

Por se tratar de dados públicos e sem identificação de seres humanos, não foi necessária a submissão do projeto do estudo a um Comitê de Ética em Pesquisa, sendo resguardado o direito da divulgação das fontes.

\section{Resultados}

A cobertura da TAN no Brasil entre os usuários do SUS foi estimada em $7,1 \%$ no ano de 2008 , passando a $10,5 \%$ em $2009,15,4 \%$ em 2010 e $21,8 \%$ em 2011 , caracterizando um crescimento de $208 \%$ no período. A cobertura estimada para cada UF está descrita na Tabela 1. Verificou-se que, das 27 UF, seis não apresentaram dados referentes aos procedimentos autorizados do SIA-SUS em 2008, cinco em 2009, uma em 2010 e duas em 2011. Apenas o Acre não apre- sentou dados para todos os anos no período.

Amapá, Mato Grosso do Sul e Paraíba foram os Estados que apresentaram as maiores estimativas de cobertura da TAN para usuários do SUS em 2008 e 2009. Em 2010 permaneceram nas três primeiras classificações o Mato Grosso do Sul, seguido da Paraíba e do Rio Grande do Sul. Já em 2011 o Rio Grande do Sul ocupa a primeira posição no nível de cobertura, seguido por Paraná e Mato Grosso do Sul. No período, destacou-se o crescimento de $432 \%$ observado no Rio Grande do Sul (11,3\% em 2008; $60,1 \%$ em 2011), e a redução da estimativa da cobertura no Amapá (79,4\% em 2008; 39,9\% em 2011).

No período de 2008-2011, houve crescimento da cobertura da TAN para os usuários do SUS no território nacional com especificidades para cada UF (Figura 1). No entanto, mesmo em 2011, 18 UF tiveram cobertura estimada inferior a $25 \%$. Consideradas as grandes regiões geográficas, todas tiveram crescimento da cobertura da TAN no período, destacando-se a evolução da cobertura estimada para as Regiões Sul (VPP $=386 \%)$ e Nordeste (VPP $=234 \%$ ) (Figura 2). As maiores coberturas da TAN no país em 2011 foram identificadas nas Regiões Sul $(54,4 \%)$ e Sudeste $(22,7 \%)$, e a menor, de apenas $11,7 \%$, na Região Nordeste. Mesmo em 2011, foi identificada ampla variação da cobertura dentre as UF pertencentes a uma mesma região, com exceção da Região Sul, onde todas superaram o patamar de $25 \%$ na cobertura da TAN. Considerando-se todas as UF com dados que permitiram estimar a cobertura em 2011, essa diferença pode ser representada por Mato Grosso do Sul $(51,4 \%)$ e Mato Grosso $(5,5 \%)$ na Região CentroOeste, Amazonas $(47,7 \%)$ e Rondônia $(4,1 \%)$ na Região Norte, Paraíba (48,9\%) e Pernambuco (4,9\%) na Região Nordeste, e São Paulo (26,3\%) e Espírito Santo $(4,2 \%)$ na Região Sudeste.

\section{Discussão}

No período de 2008 a 2011 houve um crescimento contínuo da cobertura da TAN para os usuários do SUS no país, tendo triplicado em quatro anos. No entanto, mesmo a cobertura estimada para o último ano do período, de $21,8 \%$, ainda é baixa, muito inferior à recomendada pelo Joint Committee on Infant Hearing - com o qual o Comusa, Comitê Multiprofissional em Saúde Auditiva do Brasil, é consoante - de pelo menos $95 \%$, para que então possa assumir um caráter de universalidade.12,13 Não assumindo tal caráter, também não contempla um dos princípios doutrinários do SUS, o da universalidade do acesso à saúde. 14 


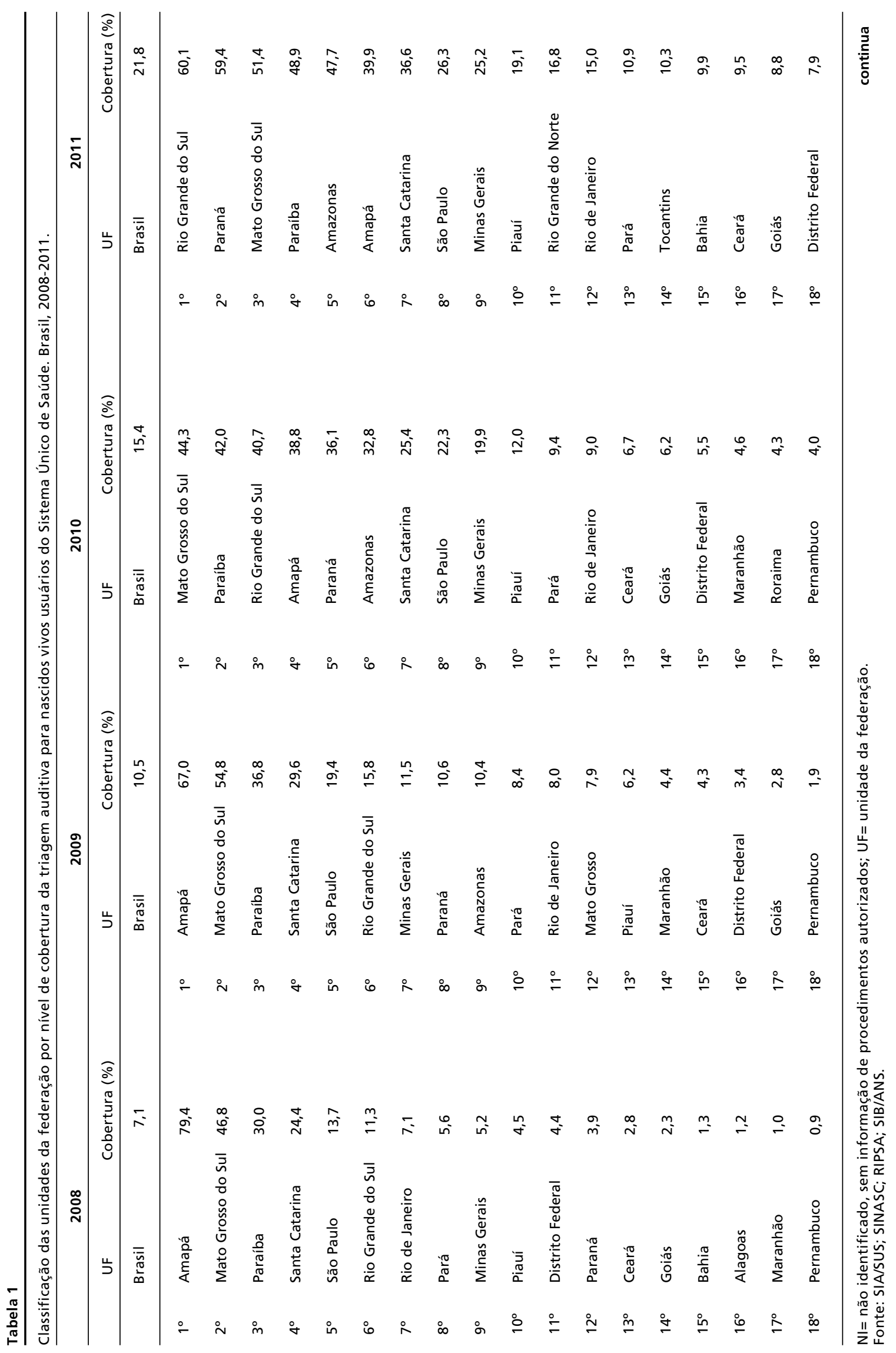




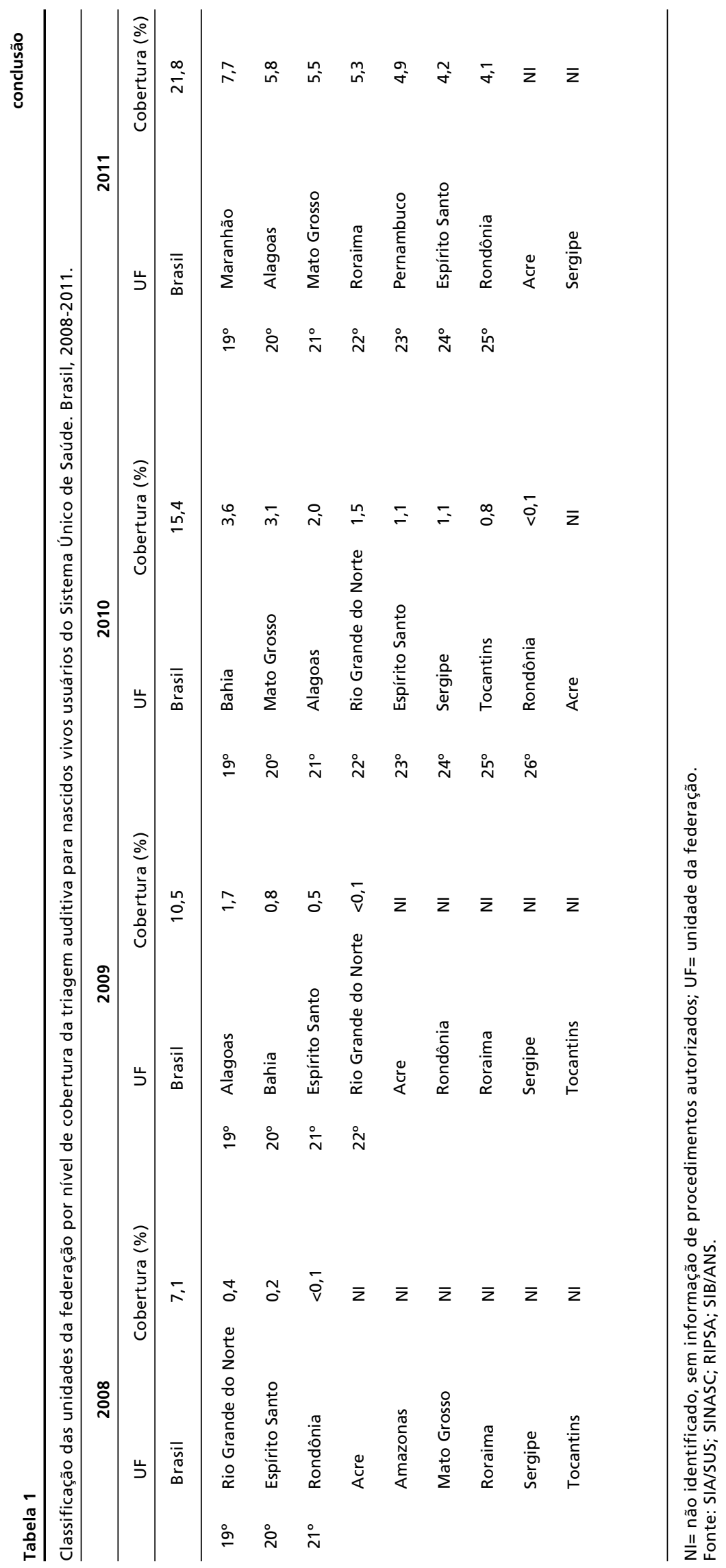


Mapa da cobertura da triagem auditiva para nascidos vivos usuários do Sistema Único de Saúde por unidade da federação no período. Brasil, 2008-2011.

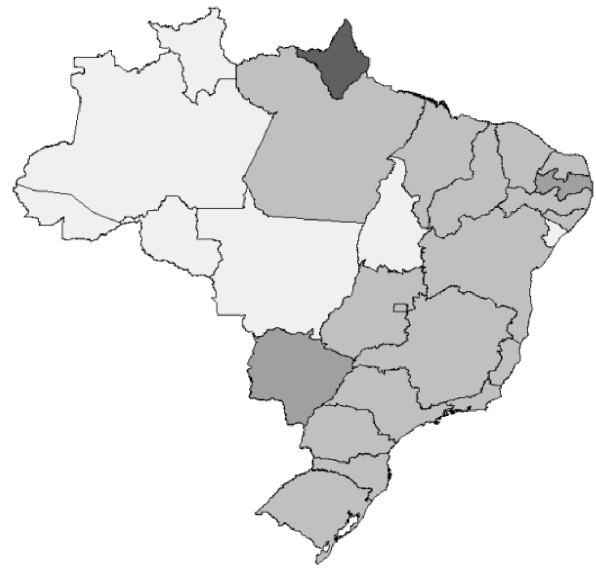

2008

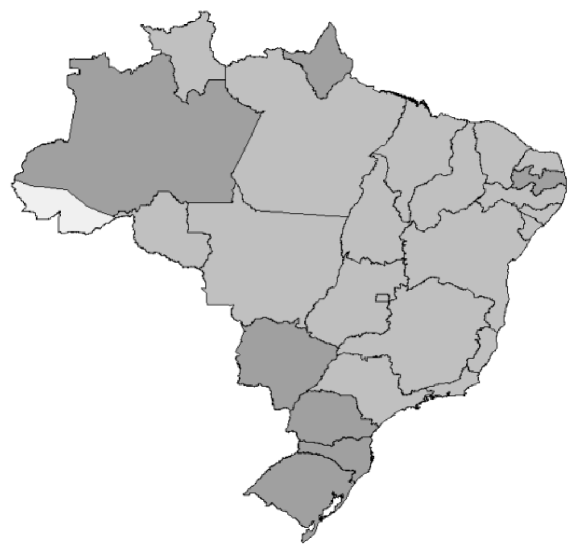

2010

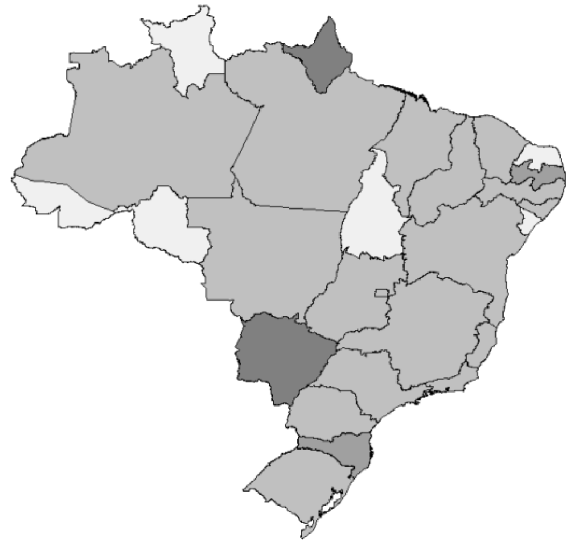

2009

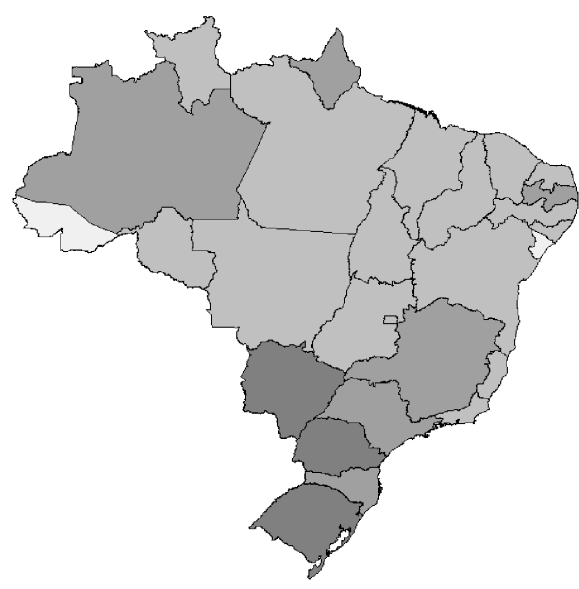

2011

Cobertura \%

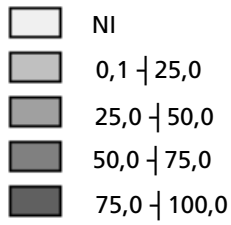

$\mathrm{NI}=$ não identificado, sem informação de procedimentos autorizados, ou com cobertura estimada $<0,1 \%$. Fonte: SIA/SUS; SINASC; RIPSA; SIB/ANS. 
Cobertura da triagem auditiva para nascidos vivos usuários do Sistema Único de Saúde por ano e região geográfica, e crescimento no período. Brasil, 2008-2011.

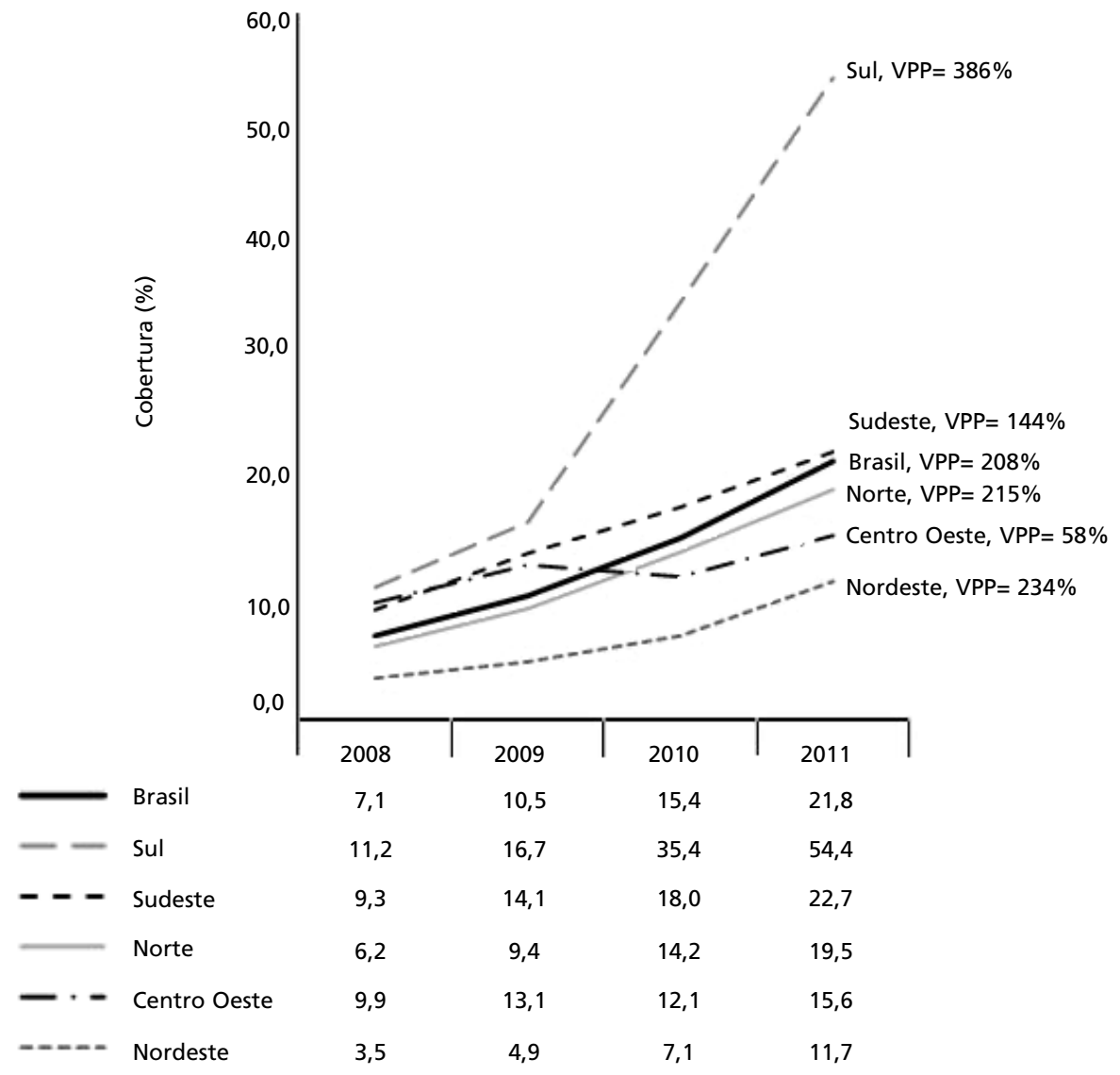

$\mathrm{VPP}=$ variação proporcional percentual: evolução da cobertura no período. Fonte: SIA/SUS; SINASC; RIPSA; SIB/ANS.

Assim, considerando-se as bases de dados disponíveis nos sistemas de informação do país, estima-se que $78,2 \%$ dos nascidos vivos que dependem do SUS não realizaram a TAN em 2011 Essa realidade contrasta com a obrigatoriedade da realização do exame de emissões otoacústicas para TAN, em território nacional, estabelecida por lei sancionada em agosto do ano anterior, 2010.11 Contrasta também com o cenário internacional, como na Inglaterra, onde desde 2006 um programa de TANU é ofertado pelo sistema de saúde, alcançando, já no primeiro ano de implantação (2006-07), cobertura de 94,6\%, evoluindo para 98,3\% em 2010-11.15 Nos Estados Unidos, ainda em 2003 , a meta de $95 \%$ de cobertura foi alcançada por
$76 \%$ dos Estados com legislação para a TANU, e por $26 \%$ dos Estados sem a lei. 16 Embora haja evidências de que a legislação é uma estratégia efetiva para o sucesso da TANU, outros fatores podem ter influência importante, como as colaborações institucionais e o financiamento público.

A expectativa é de que a TAN no Brasil torne-se universal, estratégia considerada viável e factível para detecção precoce da deficiência auditiva mesmo em países em desenvolvimento. ${ }^{17}$ No entanto, a adequação da rede de saúde e a garantia do financiamento são fatores que merecem atenção. É provável que a universalidade para a TAN demore a ser alcançada, assim como em outros programas de triagem neonatal no país, como ocorreu com o teste 
do pezinho, obrigatório por lei desde 1990, mas que apenas após dez anos atingiu cobertura de 55\%, sendo que das 27 UF, 10 ainda tinham menos de 30\% dos seus nascidos vivos submetidos à triagem. ${ }^{18}$

Em 2001, foi criado o Programa Nacional de Triagem Neonatal (PNTN) para aprimorar a Triagem Neonatal no Brasil e adotar medidas que possibilitassem o avanço de sua organização e regulação. Entre elas, determinou a implantação de Serviços de Referência em Triagem Neonatal / Acompanhamento e Tratamento de Doenças Congênitas, estabelecendo ações que integrassem todos os níveis de assistência. ${ }^{19}$ Com o PNTN houve um aumento significativo da cobertura e uma melhora na estratégia da Triagem Neonatal como Programa de Saúde Pública no Brasil. Entre 2001 e 2005, houve um aumento na cobertura da triagem neonatal (fenilcetonúria e hipotireoidismo congênito) para cerca de $80 \%$ dos nascidos vivos no país, com $74 \%$ dos Estados apresentando cobertura superior a 70\%.20 Porém, também foram detectadas desigualdades regionais nessa cobertura, com a porção Sul-Sudeste alcançando uma fase mais avançada de implantação do PNTN em relação às demais regiões, ${ }^{21}$ assim como observado neste estudo para a TAN

Evidências da desigualdade observada na cobertura da TAN no país foram identificadas nos dois níveis: inter-regional e intrarregional. Enquanto algumas regiões obtiveram crescimento acentuado na cobertura no período, como a Região Sul, em outras a evolução foi bem modesta, como na Região Centro-Oeste. Embora a Região Nordeste tenha demonstrado o segundo maior crescimento da cobertura da TAN no período, isso ocorreu porque a cobertura do primeiro ano da análise (2008), tomado como referência para a estimativa do crescimento, foi muito baixa, de apenas 3,5\%. Ressalta-se que a Região Nordeste apresentou a menor cobertura dentre as regiões para todos os anos no período 2008-2011. Desigualdades intrarregionais importantes puderam ser detectadas em todas as regiões, com exceção da Região Sul, na qual há maior uniformidade, com as suas três UF com cobertura estimada dentre as sete maiores do país. A ausência de informações no SIA-SUS sobre a realização do procedimento em determinadas UF foi mais comum no início do período, mas ainda presente em 2011, e não ocorreu nas Regiões Sul e Sudeste. Diferenças na oferta da TAN para usuários SUS observadas no país, manifestadas inter e intrarregionalmente, são o resultado de desigualdades sociais historicamente construídas, concretizadas especialmente na deficiência do acesso e na configuração inadequada das ações e serviços de saúde, 22 e refletem as dispari- dades regionais no nível de desenvolvimento social e econômico, principalmente quando se compara a porção Centro-Norte-Nordeste com a Sul-Sudeste. ${ }^{23}$

Dados da Organização Pan-Americana de Saúde (OPAS) 24 mostram que há desigualdades de distribuição na oferta dos recursos humanos no SUS. Apesar dos investimentos do SUS na tentativa de reduzir as desigualdades no acesso, o consumo de outras modalidades de assistência continua alto, e contribui para reforçar as desigualdades. A distribuição dos serviços de saúde auditiva de média e alta complexidade concentra-se majoritariamente na rede privada e filantrópica, com apenas $34 \%$ dos serviços na rede pública de saúde, com maior concentração nas Regiões Sudeste e Sul. ${ }^{25}$ Esse cenário pode contribuir para a compreensão das desigualdades na cobertura da TAN observadas neste estudo.

De modo semelhante à TAN, foram verificadas acentuadas desigualdades regionais no acesso aos serviços de saúde para tratamento odontológico no Brasil.26 Nas capitais das Regiões Sul e Sudeste foram identificadas menores proporções de falta de acesso, bem como uma maior utilização dos serviços privados, especialmente em comparação com as capitais das Regiões Norte e Nordeste. Os autores discutem a relação com o desequilíbrio na distribuição de profissionais no país, que pode favorecer a porção Sul-Sudeste e, de forma paradoxal, nas regiões mais pobres estão concentradas as maiores demandas de saúde bucal, demonstrando que os profissionais estão em menor quantidade nos locais onde existem maiores necessidades. $26 \mathrm{O}$ fonoaudiólogo é o profissional responsável pelo procedimento da TAN. Uma distribuição desigual, aliada a um quadro insuficiente de profissionais na rede pública de saúde, pode ser outro fator que se reflete nas baixas estimativas de cobertura da TAN no Brasil, e nas disparidades observadas. É plausível supor que os diversos fatores que se relacionam com as desigualdades no acesso à saúde no país se reproduzam para a saúde auditiva infantil.

As discrepâncias regionais observadas neste estudo podem também estar relacionadas ao impacto positivo da promulgação de leis municipais e estaduais, entre 1998 e 2009, que tornaram obrigatória a realização da TAN em nível local. Dos 20 municípios que já tinham a TAN regulamentada por lei, 15 eram da Região Sudeste, três da Região Sul, uma da Região Centro-Oeste, no Mato Grosso do Sul, e uma da Região Nordeste. As UF que tinham legislações sobre a TAN eram Paraná, Distrito Federal, Pernambuco e São Paulo, onde especificamente já havia lei que tratava da saúde auditiva infantil, 
embora não especificasse a realização da TAN.27-29 No entanto, mesmo em UF onde previamente ao período deste estudo já havia lei estabelecendo a obrigatoriedade da TAN, algumas apresentaram cobertura estimada muito inferior à recomendada. Um desses casos é o Estado do Pernambuco, onde a TAN é obrigatória desde 2001, embora em 2011 tenha apresentado cobertura estimada de apenas 4,9\%. Assim, percebe-se que somente a existência da lei não é suficiente para que seu cumprimento se efetive. É necessário que sejam garantidos os instrumentos e recursos necessários para viabilizar a real implantação de um Programa Nacional de Triagem Auditiva Neonatal. Casos bem sucedidos da implementação da TANU em municípios ou UF, como no caso do Rio Grande do Sul cujos dados sugerem franca evolução da cobertura no período do estudo, podem ser explorados para identificação de fatores associados ao bom desempenho e de estratégias para superação de barreiras.

A Lei Federal $n^{\circ}$ 12.303, promulgada em 2010, tornou obrigatória a realização das EOA em todas as maternidades e hospitais, gratuitamente, em todas as crianças nascidas em suas dependências. ${ }^{11}$ A lei em nível federal foi um grande avanço, e o seu cumprimento é uma necessidade, pois coloca em perspectiva a vigilância epidemiológica da perda auditiva em neonatos, permitindo gerar conhecimento que contribuirá para um melhor planejamento em saúde e para a avaliação do impacto de intervenções. Por meio da vigilância, o conhecimento da realidade da condição de saúde auditiva dos neonatos nos níveis municipal, estadual e federal, poderá subsidiar o planejamento de programas e ações de promoção da saúde auditiva, de forma descentralizada e democrática, a partir também das necessidades locais. Neste cenário, será favorecido o planejamento de ações de prevenção da deficiência auditiva e de suas consequências, da oferta e acesso a procedimentos avançados de diagnóstico e de intervenção para garantir o melhor desenvolvimento das potencialidades do indivíduo, contribuindo para seu bem estar e inserção mais efetiva na sociedade.

Entretanto, ainda existem controvérsias na compreensão da lei. Dentre elas, destacamos: o não estabelecimento de prazos para cumprimento e a indefinição quanto às fontes de financiamento. Estas questões podem interferir na sua aplicabilidade e/ou efetivação. É importante que se estabeleça uma rede de saúde auditiva cuja gestão desempenhe ativamente o seu papel, assegurando assim o desenvolvimento adequado de cada programa integrado à rede, sendo essencial um programa nacional que contemple desde a triagem auditiva universal, seguida por diagnóstico, intervenção e acompanhamento dos usuários. 30 Assim, espera-se que ajustes sejam feitos por parte do governo federal, a fim de esclarecer as lacunas ainda existentes e normatizar a execução na forma que estabelece a lei. Um passo nessa direção foi o estabelecimento das diretrizes de atenção da TAN no Brasil, em 2012.6 A expectativa para os próximos anos é de crescimento da cobertura da TAN em todo o país, processo que poderá ser mais ágil com maior clareza das normas e maior participação do controle social, permitindo alcançarmos em menor tempo o patamar de $95 \%$ de cobertura, a triagem auditiva neonatal universal no Brasil.

Embora o uso de dados secundários, de domínio público, permita sua disponibilidade imediata, traduzindo-se em agilidade para a análise, a demora na atualização dos dados públicos de saúde foi um fator limitante do estudo, impedindo uma análise de maior abrangência temporal. Outra limitação, relacionada ao uso dos dados dos Sistemas de Informação em geral, é o sub-registro. Neste sentido, foi adotado o fator de correção da RIPSA para ajuste dos dados do SINASC, reduzindo vieses nas estimativas de cobertura. No SIA-SUS, além do subregistro, pode haver valores superestimados. O SIASUS é base para o faturamento dos procedimentos realizados pelas unidades especializadas do SUS, o que pode ter levado a vieses, elevando algumas estimativas de cobertura, em consequência do registro de um número maior de procedimentos do que os efetivamente realizados. Mudanças abruptas na cobertura estimada em anos subsequentes podem sugerir problemas dessa ordem. O registro no SIASUS depende de um protocolo regular de preenchimento de fichas de procedimentos realizados, e alimentação do sistema. Assim, o nível de organização dos serviços de saúde pode influenciar positiva ou negativamente nesse processo, e a ausência de dados, como observado em determinadas UF, não significa necessariamente que nenhum exame foi realizado. Outra questão que merece cuidado na interpretação é a representatividade da cobertura para o território da UF, em vista dos diferentes graus de urbanização, o que pode elevar a cobertura em UF mais urbanizadas, e mascarar possíveis desigualdades de acesso intra-estaduais. Por fim, o parâmetro utilizado para estimar a parcela dos usuários do SUS, a partir de dados do SIB, apresenta limitações, entre elas, a possibilidade de uma beneficiária não ter seu parto coberto pelo plano de saúde suplementar e então realizar o procedimento da TAN no SUS, ou de um mesmo beneficiário possuir mais de um plano de saúde. Essas duas condições poderiam elevar a 
estimativa de cobertura da TAN para usuários SUS. As limitações não inviabilizam o estudo nem reduzem sua importância, mas precisam ser conhecidas e consideradas na compreensão dos resultados. Questões como o sub-registro e valores superestimados podem, inclusive, ser adotadas como objeto de estudo e análise nas próprias UF.

Este é o primeiro estudo que avaliou a cobertura nacional da TAN no Brasil, e avança com a divulgação de um quadro descritivo inicial, porém representativo, de vigilância da realização da triagem auditiva dos nascidos vivos usuários do SUS no país. Ressalta-se que para o estudo foram produzidas esti-

\section{Referências}

1. Tan CQ, Dong WD, Guo L, Huang H, Wang DY. Auditory function in women with autoimmune inner ear diseases and their offspring. Int J Pediatr Otorhinolaryngol. 2009; 73: 1702-11

2. Oliveira P, Castro F, Ribeiro A. Surdez infantil. Rev Bras Otorrinolaringol. 2002; 68: 417-23.

3. World Health Organization Deafness and hearing loss - Fact Sheet No. 300. [acesso em 5 mai 2014]. Disponível em http://www.who.int/mediacentre/factsheets/fs300/en/index. $\mathrm{html}$

4. Yoshinaga-Itano C. Benefits of early intervention for children with hearing loss. Otolaryngol Clin North Am. 1999; 32: 1089-102.

5. Joint Committee on Infant Hearing. Joint Committee on Infant Hearing 1994 Position Statement. [acesso em 2 nov 2011]. Disponível em: http://www.jcih.org/JCIH1994.pdf

6. Brasil. Ministério da Saúde. Secretaria de Atenção à Saúde Departamento de Ações Programáticas Estratégicas. Diretrizes de Atenção à Triagem Auditiva Neonatal. Brasília, DF; 2012

7. Vieira ABC, Macedo LR, Gonçalves DU. O diagnóstico da perda auditiva na infância. J Pediatr. 2007; 29: 43-9.

8. Grupo de Apoio à Triagem Auditiva Neonatal Universal Programas de triagem auditiva neonatal [acesso em 2 nov 2011]. Disponível em: http://www.gatanu.org

9. Sanches RM, Ciconelli RM. Conceitos de acesso à saúde. Rev Panam Salud Publica. 2012; 31 (3): 260-8.

10. Brasil. Ministério da Saúde. Secretaria de Gestão Estratégica e Participativa. Departamento de Apoio à Gestão Participativa. Políticas de promoção da equidade em saúde. Brasília, DF; 2013.

11. Brasil. Lei $\mathrm{n}^{\circ} 12.303$, de 2 de agosto de 2010. Dispõe sobre a obrigatoriedade de realização do exame denominado Emissões Otoacústicas Evocadas. Diário Oficial da União [DOU]. Brasília, DF 2 ago 2010.

12. Joint Committee on Infant Hearing. Year 2000 position statement: principles and guidelines for early hearing detection and intervention programs. J Pediatr. 2000; 106: 798817

13. Lewis DR, Marone SAM, Mendes BCA, Cruz OLM, mativas a partir de bases de vários sistemas de informação de domínio público do país, e que esses sistemas são bases dinâmicas disponibilizadas pelo governo de várias nações com o propósito da vigilância, e assim permitem o monitoramento continuado das questões relativas à saúde, sendo também úteis para avaliar o impacto de leis e diretrizes governamentais. A metodologia proposta neste estudo pode ser reproduzida para gerar informações periódicas sobre a cobertura e avaliação da implementação da TAN nos municípios ou regionais de saúde, caso não estejam disponíveis dados primários, auxiliando o planejamento em saúde em nível local.
Nóbrega M. Comitê multiprofissional em saúde auditiva: COMUSA. Rev Bras Otorrinolaringol. 2010; 76: 121-8.

14. Brasil. Lei ${ }^{\circ} 8.080$, de 19 de setembro de 1990. Dispõe sobre as condições para promoção, proteção e recuperação da saúde, a organização e o funcionamento dos serviços correspondentes e dá outras providências. Diário Oficial da União [DOU]. Brasília, DF 19 set 1990.

15. UK National Screening Committee. Annual Report. NHS Newborn Hearing Screening Programme 2010/2011. Annual Report and 2009-2010 Data Report. MRC Hearing and Communication Group, London, UK; 2012.

16. Green DR, Gaffney M, Devine O, Grosse SD. Determining the effect of newborn hearing screening legislation: an analisys of state hearing screening rates. Public Health Reports. 2007; 122 (2): 198-205.

17. Olusanya BO, Swanepoel de W, Chapchap MJ, Castillo S, Habib H, Mukari SZ, Martinez NV, Hung-Ching Lin, Bradley McPherson. Progress towards early detection services for infants with hearing loss in developing countries. BMC Health Serv Res. 2007; 7: 14.

18. Brasil. Ministério da Saúde. Diagnóstico situacional do programa nacional de triagem neonatal. 2011. [acesso em 22 dez 2011]. Disponível em: http://portal.saude.gov. br/portal/arquivos/pdf/pntn_triagemneonatal_.pdf

19. Brasil. Portaria $n^{\circ} 822$, de 06 de junho de 2001. Institui no âmbito do Sistema Único de Saúde o Programa Nacional de Triagem Neonatal/PNTN. Diário Oficial da União [DOU]. Brasília, DF 6 jun 2001

20. Carvalho TM, Santos HP, Santos ICGP, Vargas PR, Pedrosa J. Newborn screening: a national public health programme in Brazil. J Inherit Metab Dis. 2007; 30: 615.

21. Brasil. Ministério da Saúde. Triagem neonatal. Localização dos serviços. [acesso em 22 dez 2011]. Disponível em: http://portal.saude.gov.br/portal/saude/area. cfm?id area $=1061$

22. Luiz OC, Heimann LS, Boaretto RC, Pacheco AG, Pessoto UC, Ibanhes LC, Castro IEN, Kayano J, Junqueira V, Rocha JL, Cortizo CT, Telesi Jr E. Diferenciais intermunicipais de condição de vida e saúde: construção de um indicador composto. Rev Saúde Pública. 2009; 43 (1): 115-22. 
23. Rodrigues CG, Amaral PVM, Simões RF. Rede Urbana da Oferta de Serviços de Saúde: uma análise multivariada macro regional - Brasil, 2002. Rev Desenvolv Econ. 2007 9: 83-92.

24. Nunes A, Santos JRS, Barata RB, Vianna SM. Medindo as desigualdades em Saúde no Brasil: uma proposta de monitoramento. Brasília: Organização Pan-Americana da Saúde, Instituo de Pesquisa Econômica Aplicada; 2001.

25. Andrade CL, Fernandes L, Ramos HE, Mendes CMC, Alves CAD. Programa Nacional de Atenção à Saúde Auditiva: avanços e entraves da saúde auditiva no Brasil. Rev Ciênc Med Biol. Salvador: 2013; 12: 404-10.

26. Peres MA, Iser BPM, Boing AF, Yokota RTC, Malta DC, Peres KG. Desigualdades no acesso e na utilização de serviços odontológicos no Brasil: análise do Sistema de Vigilância de Fatores de Risco e Proteção para Doenças Crônicas por Inquérito Telefônico (VIGITEL 2009). Cad Saúde Pública. 2012; 28 (Supl.): S90-S100.
27. Tochetto TM, Vieira EP. Legislação brasileira sobre triagem auditiva neonatal. Carapicuíba: Pró Fono; 2006.

28. Conselho Regional de Fonoaudiologia $2^{\text {a }}$ Região. Legislação. [acesso em 22 dez 2011]. Disponível em: http://www.fonosp.org.br/legislacao/

29. Conselho Regional de Fonoaudiologia $8^{\mathrm{a}}$ Região. Leis e Decretos. Lei 5.960, de 02 set 2009. [acesso em $22 \mathrm{dez}$ 2011]. Disponível em: http://crefono8.gov.br/?ui=legislacao\&uiui $=$ leis \& tipo $=4$

30. Alvarenga KF, Bevilacqua MC, Costa AO, Martinez MAN Estado atual da saúde auditiva neonatal no Brasil: políticas públicas e evidências científicas. In: Bevilacqua MC, Martinez MAN, Balen AS, Pupo AC, Reis ACM, Frota S, organizadores. Saúde auditiva no Brasil: políticas, serviços e sistemas. São José dos Campos: Pulso Editorial; 2010. p. 97-117.

Recebido em 26 de dezembro de 2013

Versão final apresentada em 12 de junho de 2014

Aprovado em 18 de julho de 2014 